\title{
Antiaging and Antioxidant Bioactivities of Asteraceae Plant Fractions on the Cellular Functions of the Yeast Schizosaccharomyces pombe
}

\author{
Rika Indri Astuti $\mathbb{D}^{1,2}$ Muhammad Eka Prastya $\mathbb{D}^{3},{ }^{3}$ Irmanida Batubara $\mathbb{D}^{2,4}$ Eka Budiarti, ${ }^{4}$ \\ and Aulia Ilmiyawati ${ }^{4}$ \\ ${ }^{1}$ Department of Biology, Faculty of Mathematics and Natural Sciences, IPB University, IPB Dramaga Campus, Bogor, \\ West Java 16680, Indonesia \\ ${ }^{2}$ Tropical Biopharmaca Research Center, IPB University, Jl. Taman Kencana No. 3, Bogor, West Java 16128, Indonesia \\ ${ }^{3}$ Research Center for Chemistry, Indonesian Institute of Sciences (LIPI), National Research and Innovation Agency (BRIN), \\ Kawasan PUSPITEK, Serpong, Tangerang Selatan, Banten 15314, Indonesia \\ ${ }^{4}$ Department of Chemistry, Faculty of Mathematics and Natural Sciences, IPB University, IPB Dramaga Campus, Bogor, \\ West Java 16680, Indonesia
}

Correspondence should be addressed to Irmanida Batubara; ime@apps.ipb.ac.id

Received 14 April 2021; Revised 11 August 2021; Accepted 7 September 2021; Published 20 September 2021

Academic Editor: Mounir Tilaoui

Copyright (c) 2021 Rika Indri Astuti et al. This is an open access article distributed under the Creative Commons Attribution License, which permits unrestricted use, distribution, and reproduction in any medium, provided the original work is properly cited.

\begin{abstract}
Research on antioxidants has been gaining worldwide attention because of their essential applications for medicinal purposes. In this study, we conducted bioprospecting of six Asteraceae plants as the source of antiaging and antioxidant agents. Water and chloroform fractions from Ageratum conyzoides L., Dichrocephala integrifolia (L.f.) Kuntze, Galinsoga parviflora (Cav.), Mikania micrantha Kunth, Sphagneticola trilobata (L.) Pruski, and Synedrella nodiflora L. were collected and assayed for their in vitro antioxidant activities and potential antiaging properties using the yeast Schizosaccharomyces pombe as the model organism. Based on the in vitro assay, the water fractions of $S$. trilobata showed a strong antioxidant activity. Interestingly, all treatment solutions promoted the stress tolerance phenotype of $S$. pombe to strong $\mathrm{H}_{2} \mathrm{O}_{2}$-induced oxidative stress conditions. Moreover, compared with the treatments without plant extract/fraction, all extract and fraction treatments, except the chloroform fractions of A. conyzoides, promoted yeast cell longevity. Strong induction of mitochondria activity was found following the treatments with the extracts and fractions of $S$. nodiflora, D. integrifolia, and M. micrantha and likely mimicked the calorie restriction-induced lifespan. Interestingly, S. nodiflora water fractions significantly upregulated the mRNA transcripts of the Pap1-mediated core environmental stress response, namely, cttl gene in S. pombe. These data indicated that the fractions of Asteraceae plants had potential antioxidant and antiaging activities through various cellular modulations. S. nodiflora water fraction has been shown to have antioxidant and antiaging activities in $S$. pombe, by modulating stress tolerance response, inducing mitochondrial activity, and increasing the cttl gene expression. Compounds analysis identified that $S$. nodiflora water fraction contained some primarily compounds including oxyphyllacinol, valine, and sugiol.
\end{abstract}

\section{Introduction}

Free radical accumulation has been known to induce oxidative stress conditions, which further damage cellular functions $[1,2]$. Through the activation of enzymatic and nonenzymatic antioxidants, the human body has complex defense systems against these damaging effects of free radicals $[3,4]$. As the body ages or after intensive exposure to exogenous free radical sources, the cellular defense systems may be compromised and cause severe oxidative stress conditions, which often culminate in the development of degenerative diseases [5]. Exogenous antioxidants, which are 
also known as dietary antioxidants, are often used to reduce the damaging effects of free radicals. Therefore, the intake of exogenous antioxidants may compensate for the inadequacy of cellular antioxidant activity in combating oxidative stress conditions [6].

Plants have been known as the major sources of antioxidant compounds, most of which are phenolic $[7,8]$. Among the plant families, Asteraceae is the second largest family and is the largest family of flowering plants in the kingdom Plantae. Therefore, exploration of plants that belong to the Asteraceae family is widely open, especially for those considered as weed. In fact, weed has been the most subjected plant for intensive control in farm fields; however, their value for bioprospecting may not have been properly explored. Previous studies have revealed the medicinal value of Asteraceae plants in vitro [9-12] and suggested the potential use of plant extract as dietary antioxidants. However, until today, analyses of the antioxidant activities of Asteraceae-derived extracts in modulating cellular systems have been very limited. Such information is essential for the comprehensive evaluation of the antioxidant properties of the extracts, especially in revealing the mechanism of actions at the cellular level, and the application of these extracts as dietary antioxidants for daily use.

In this study, we analyzed the potential antioxidant activity of the weed plant extracts of Ageratum conyzoides L., Dichrocephala integrifolia (L.f.) Kuntze, Galinsoga parviflora (Cav.), Mikania micrantha Kunth, Sphagneticola trilobata L. Pruski, and Synedrella nodiflora L. The effect of those extracts at the cellular level was evaluated using the yeast Schizosaccharomyces pombe as the model organism. Our previous study has already reported the antioxidant activity of the water extract of each plant sample [13]. Therefore, in this study, we further determined the potential antioxidant properties of the water and chloroform fractions, as well as the water extract of each plant, based on the capability of promoting yeast longevity. Oxidative stress has been known to be a major factor in cellular aging. Therefore, the application of exogenous antioxidants may inhibit cellular aging and promote longevity [14]. The molecular pathways of cellular aging between $S$. pombe and the multicellular eukaryotic organisms are homologous and involve the activities of the sirtuin $[15,16]$ and mitogen-activated protein kinase genes $[17,18]$, autophagy $[19,20]$, and mitochondria [21-23]. In addition, calorie restriction has also been implied as exogenous treatment that induces the complex longevity pathway which was conserved from yeast to human $[23,24]$. Therefore, in this research, we aimed to determine the mechanism of actions of particular plant extracts by evaluating their regulatory functions on the activities of the mitochondria and the oxidative stress response genes.

\section{Materials and Methods}

2.1. Samples. Six species of Asteraceae that easily grow in Indonesia were collected from the Conservation and Cultivation Unit of Tropical Biopharmaca Research Center Bogor, West Java, Indonesia (GPS location: $6^{\circ} 35^{\prime} 17.8^{\prime \prime} \mathrm{S}$ $106^{\circ} 48^{\prime} 05.4^{\prime \prime} \mathrm{E}$ ). Each species was determined by a botanist in the Indonesian Institute of Sciences (LIPI) Biology at Cibinong, Jakarta, Indonesia. Voucher specimens of the samples were deposited in the Tropical Biopharmaca Research Center, IPB University, Indonesia. The list of samples and voucher specimen numbers is shown in Table 1. For this study, the aerial plant parts, including twigs and leaves, were used.

2.2. Yeast Strain and Growth Conditions. We used the yeast strain of S. pombe ARC039 (h-leu1-32 ura4-294) to investigate the antiaging effects of the extracts at the cellular level [25]. For the viability assay, the yeast cells were grown in Yeast Extract with Supplements (YES) medium with 3\% glucose and in Edinburgh Minimal Medium (EMM) with $0.5 \%$ glucose as the negative and positive controls, respectively [26].

2.3. Extraction and Fractionation. All samples were extracted with water $(1 \mathrm{~g}$ dried sample to $10 \mathrm{~mL}$ water) at room temperature for 24 hours. Thereafter, the filtrate was separated from the residue and was concentrated by a rotary evaporator to get the crude extract. Then, the crude extract was separated by liquid-liquid extraction using chloroform, in order to get the water and chloroform fractions. Each fraction was concentrated using a rotary evaporator.

2.4. Antioxidant Assay. The antioxidant activities of the fractions were determined using the radicals of 2,2'diphenylpicryl hydrazyl (DPPH, Sigma-Aldrich) and 2,2' azino-bis(3-ethylbenzothiazoline-6-sulfonic acid) (ABTS, Carbosynth Ltd., UK). The anti-DPPH scavenging activity was determined using spectrophotometry assay, as described elsewhere [27]. The fractions $(100-1500 \mu \mathrm{g} / \mathrm{mL})$ were diluted with ethanol (96\%), reacted with DPPH, and incubated for 30 minutes. Thereafter, the absorbance of the solution was determined at $514 \mathrm{~nm}$. The anti-ABTS activity was determined using a spectrophotometric method [27]. Briefly, $7 \mathrm{mM}$ ABTS was oxidized by potassium peroxide sulfate to produce the ABTS radical, which was then reacted with a fraction solution $(10 \mathrm{mg} / \mathrm{mL}$ in $96 \%$ ethanol). The absorbance of the solution was measured at $734 \mathrm{~nm}$. Both DPPH and ABTS assays were conducted using a multiscan ELISA microtiter plate reader (EPOC Biotech, USA). The ability of the fractions to scavenge DPPH and ABTS radicals was calculated as $\%$ inhibition by the same following equation:

$$
\% \text { inhibition }=1-\left\{\frac{(\text { Abs. sample }- \text { Abs. blank })}{\text { Abs. control }- \text { Abs. blank }}\right\} \times 100 \text {. }
$$

Here, Abs.sample is the absorbance of DPPH/ABTS radicals + fractions or positive control (ascorbic acid/Trolox); Abs.control and Abs.blank are the absorbance of the DPPH/ABTS radicals + ethanol and the absorbance of ethanol, respectively. As for DPPH activity, the results are expressed as the inhibitory concentration of $50 \%\left(\mathrm{IC}_{50}\right)$, whereas ABTS activity is reported in mg Trolox equivalent (TE)/g sample. 
TABLE 1: Species, local name, and voucher specimen number of all samples used in this study.

\begin{tabular}{lccc}
\hline No. & Species & Local name & Voucher specimen number \\
\hline 1 & Ageratum conyzoides L. & Bandotan & BMK0176092016 \\
2 & Dichrocephala integrifolia (L.f.) Kuntze & Jukut Meurit & BMK0406102018 \\
3 & Galinsoga parviflora (cav.) & Loseh & BMK0415102018 \\
4 & Mikania micrantha Kunth & Sembung Rambat & BMK0419012018 \\
5 & Sphagneticola trilobata L. Pruski & Wedelia/Pruski & BMK0364102018 \\
6 & Synedrella nodiflora L. & Jotang Kuda & BMK0446012018 \\
\hline
\end{tabular}

2.5. Oxidative Stress Tolerance Assay. The experiment was carried out to evaluate the effect of the plant extracts/fractions in promoting cell viability against oxidative stress [28]. The yeast cells were inoculated in YES liquid medium for the treatment culture, with an initial $\mathrm{OD}_{600}$ of 0.05 . Simultaneously, different concentrations of the extracts/fractions (at concentrations ranging from 1 to 5 times the $\mathrm{IC}_{50}$ value based on the DPPH assay) dissolved in $99 \%$ DMSO (at a final concentration of 5\%) were added to the treatment culture. After incubation for 7 and 11 days with centrifugation at $120 \mathrm{rpm}$ at $30^{\circ} \mathrm{C}$, the yeast cells were streaked onto the plates with YES medium containing $5 \mathrm{mM} \mathrm{H}_{2} \mathrm{O}_{2}$. After three days of incubation at $30^{\circ} \mathrm{C}$, cell viability was observed based on the density of the colonies on the agar plate. In this assay, the yeast cells cultured in YES and EMM media, which were both supplemented with $99 \%$ DMSO (at a final concentration of $5 \%$ ), were used as negative and positive controls, respectively.

2.6. Aging Assay. Aging assay was conducted based on a previous study [29]. The yeast cells were grown in YES liquid medium containing the plant extracts/fractions. The applied concentration of the extracts/fractions was based on the oxidative stress tolerance assay (i.e., 1-5 times concentrations of $\mathrm{IC}_{50}$, based on the DPPH activity). Briefly, the yeast culture was prepared as described above in the oxidative stress tolerance assay. The yeast cells were harvested on days 7 and 11 and were serially diluted in sterile water at an initial $\mathrm{OD}_{600}$ of 1 . Next, $3 \mu \mathrm{L}$ of each dilution was spotted onto the YES agar plates and incubated for three days at $30^{\circ} \mathrm{C}$. Cell viability was evaluated qualitatively.

For further evaluation, the number of cells in the day 11 culture was quantified based on the plate count assay [30]. Day 11 cultures were harvested and serially diluted using sterile water, as described previously. Thereafter, about $100 \mu \mathrm{L}$ of the culture was spread onto the YES agar plate and incubated for three days at $30^{\circ} \mathrm{C}$. The number of cells was then enumerated. The yeast cells that grew in the EMM and YES media, which were both supplemented with $99 \%$ DMSO (at a final concentration of 5\%), were used as the positive and negative controls, respectively.

2.7. Mitochondrial Activity Assay. The mitochondrial activity and the mitochondrial membrane potential of the yeast cells were analyzed using a fluorescent probe (Rhodamine B; Sigma-Aldrich, USA) [31]. The yeast cells were cultured in YES medium supplemented with the plant extracts/fractions (with the concentration from 1 to 5 times of $\mathrm{IC}_{50}$, following the DPPH activity) at an initial $\mathrm{OD}_{600}$ of
0.05 to the log phase. Further, the yeast cells were harvested and washed with phosphate-buffered saline (PBS), which had a $\mathrm{pH}$ of 7.4, prior to suspension with Rhodamine B at a final concentration of $100 \mathrm{nM}$ on PBS. After incubation for 30 minutes, the yeast cells were washed, and mitochondrial activity was observed on a fluorescent microscope (Olympus BX51).

\subsection{Gene Expression Analysis Using Quantitative Reverse} Transcriptase-Polymerase Chain Reaction. The best concentrations of plant extracts/fractions (S. nodiflora: water extract: $8000 \mathrm{ppm}$, water fraction: $1794 \mathrm{ppm}$, and chloroform fraction: $3885 \mathrm{ppm}$ ) dissolved in $99 \% \mathrm{DMSO}$ (at a final concentration of $5 \%$ ) were supplemented onto the YES liquid medium after the addition of the yeast inoculum at an initial $\mathrm{OD}_{600}$ of 0.05 . Notably, $99 \%$ DMSO at a final concentration of $5 \%$ was used as the control for these experiments. After incubating the particular treatment for 24 hours, RNA was extracted from the yeast cells using the RNeasy Mini Kit (Qiagen, USA). Reverse transcription was done using iScript ${ }^{\mathrm{TM}} \mathrm{cDNA}$ Synthesis Kit (Bio-Rad, USA), with iScript reverse transcriptase and $500 \mathrm{ng}$ of total RNA. Quantitative reverse transcriptase-polymerase chain reaction was performed using Applied Biosystems StepOnePlus ${ }^{\mathrm{TM}}$ Instrument and Thunderbird SYBR qPCR master mix (Toyobo, Japan) as the fluorescent reporter. The thermal cycling parameters were as follows: 40 cycles, $95^{\circ} \mathrm{C}$ for 15 seconds, $55^{\circ} \mathrm{C}$ for 30 seconds, and $72^{\circ} \mathrm{C}$ for 30 seconds. The list of primers from the target genes, including act1 (housekeeping gene), pap1, ctt1, and sod2, is shown in Table 2. To measure the relative expression level, the cycle threshold $(\mathrm{Ct})$ values of all target genes were normalized to the Ct of act1.

2.9. Liquid Chromatography Quadrupole-Mass Spectrometry (LC-MS/MS). The selected fractions were analyzed using Xevo G2-XS QTof (Quadrupole Time-of-Flight) mass spectrometry instrument (Waters, USA) via an electron spray interface (ESI). Chromatographic separation conditions were performed using an LC system in the form of Ultra Performance Liquid Chromatography (UPLC)/QTof MS analytical system (Waters). Separation was achieved by stepwise gradients from $95 \%$ A $(0.1 \%$ formic acid + distilled water) and 5\% B (acetonitrile $+0.1 \%$ formic acid) to $5 \% \mathrm{~A}$ and $95 \%$ B for 16 minutes. The flow rate of the desolvation gas was set to $1000 \mathrm{~L} / \mathrm{h}$, for cone gas it was set to $50 \mathrm{~L} / \mathrm{h}$, and the source temperature was fixed to $120^{\circ} \mathrm{C}$. The capillary voltage and cone voltage were set to 2.0 and $30 \mathrm{kV}$, 
TABLE 2: Primer pairs used in the analysis of gene expression by using qRT-PCR.

\begin{tabular}{lc}
\hline Targeted genes & \multicolumn{1}{c}{ Primer sequence } \\
\hline Reference gene (act1+) & \\
Forward (F) & $5^{\prime}$ CGGTCGTGACTTGACTGACT 3' \\
Reverse (R) & $5^{\prime}$ ATTTCACGTTCGGCGGTAGT 3' \\
\hline Transcriptional factor Pap1 (pap1+) & \\
Forward (F) & $5^{\prime}$ TGGATGGCGATGTTAAGCCT 3' \\
Reverse (R) & $5^{\prime}$ GCAGCACGGTTTTGAGCTTT 3' \\
\hline Superoxide dismutase 2 (sod2+) & \\
Forward (F) & $5^{\prime}$ ATTTGGAGGGAGAGGTTGCC 3' \\
Reverse (R) & $5^{\prime}$ GATTGATGTGACCACCGCCA 3' \\
\hline Catalase (ctt1+) & \\
Forward (F) & $5^{\prime}$ TCGTGACGGCCCTATGAATG 3' \\
Reverse (R) & $5^{\prime}$ AGCAAGTGGTCGGATTGAGG 3' \\
\hline
\end{tabular}

respectively. The mass spectrometry was determined using the type of electrospray ionization (ES) Xevo G2-S QTof (Waters) with Quadrupole Time-of-Flight mass spectrometry in positive ion mode. Moreover, the accurate mass and composition for the precursor ions and fragment ions were calculated and identified using the UNIFI software library incorporated in the instrument.

2.10. Statistical Analyses. All data were represented as mean \pm SEMs $(n=3)$. The significant differences among the groups in all experiments were determined using one-way analysis of variance with $95 \%$ confidence level, followed by multiple Duncan test ranges. A p-value of less than 0.05 was considered statistically significant.

\section{Results and Discussion}

3.1. Antioxidant Activity of Plant Extracts and Fractions In Vitro. In the present study, we used water as for particular solvent to extract the primary compounds of all samples. Of note, water has been used routinely to extract active compounds from plants in the early step of making Indonesian herbal medicine, namely, "Jamu," traditionally and industrially. We suggested that the corresponding methods used in this study could obtain the various active compounds and thus would provide new valuable insight for developing "Jamu" derived from Asteraceae plant extract. In the initial study and on the basis of the in vitro antioxidant assays using DPPH and ABTS radicals, the water fraction of S. trilobata showed the highest antioxidant activity (Figures 1(a) and 1(b)). As shown in Figure $1(\mathrm{a})$, the $\mathrm{IC}_{50}$ value of $S$. trilobata water fraction (108.09 ppm) is the lowest compared to others which indicated strong activity to scavenge DPPH radicals. Of note, $\mathrm{IC}_{50}$ was defined as the concentration that could inhibit a reaction by about $50 \%$. The most active DPPH samples were those with the lowest $\mathrm{IC}_{50}$ values. On the other hand, the anti-ABTS samples were measured as the Trolox equivalent antioxidant activity (TEAC, mg Trolox equivalent/g). The most active ABTS sample was the sample with the highest value of TEAC. Together, S. nodiflora water fraction exhibited the highest value of $4.20 \mathrm{mg}$ Trolox/g fraction in the ABTS assay (Figure 1(b)). Nevertheless, the activity of the particular water fraction was not as good as that of pure ascorbic acid as the positive control.

\subsection{Antioxidant Activity of the Plant Extracts and Fractions on} Yeast Cell Viability. To determine the optimum concentrations of the plant extract and fractions for regulating the oxidative stress response in yeast cells, we tested the effects of various concentrations of the plant extract and fractions on the cell viability of yeast in $\mathrm{H}_{2} \mathrm{O}_{2}$-induced oxidative stress conditions. The concentrations of the plant extracts/fractions used in this assay were determined starting from $1 \times \mathrm{IC}_{50}$ value of DPPH activity followed by 2 up to 5 times $\mathrm{IC}_{50}$. Based on our results, treatment with all plant extracts and fractions (except A. conyzoides chloroform fraction) enhanced the stress tolerance phenotype of the yeast $S$. pombe, compared with the effects seen without treatment (control) (Table 3). In addition, those plant extracts and fractions treatment resulted in higher cell viability than that of calorie restriction treatment (Table 3). Calorie restriction treatment has been known to trigger a reduction in cellular damage induced by reactive oxygen species [32]. The effect of extracts/fractions treatments was indicated by the viability of the remaining cells that were still viable on the spot assay under the oxidative stress condition as shown in the additional figure in Table 3. This reveals the potential activity of plant extracts treatments in mimicking calorie restriction treatment even at high-calorie nutrition (3\% glucose in YES medium) used in this study.

Among the extract and fraction concentrations, the water extract of $D$. integrifolia and the water fraction of G. parviflora highly promoted cell viability against oxidative stress. However, such phenotype was observed following high concentrations of the extracts/fractions treatment $(5 \mathrm{x}$ times of the $\left.\mathrm{IC}_{50}\right)$. On the other hand, a lower value which indicated stronger activity of $3 \mathrm{x}$ times from the $\mathrm{IC}_{50}$ concentrations of both $S$. nodiflora water and chloroform fractions highly induced an oxidative stress tolerance phenotype in the yeast $S$. pombe, thereby suggesting the potential application as an exogenous antioxidant.

Our data indicated that the plant extracts/fractions induced the capability of yeast cells to cope with strong oxidative stress conditions. Previous studies revealed the potential of various extracts of plants, including rosella and 


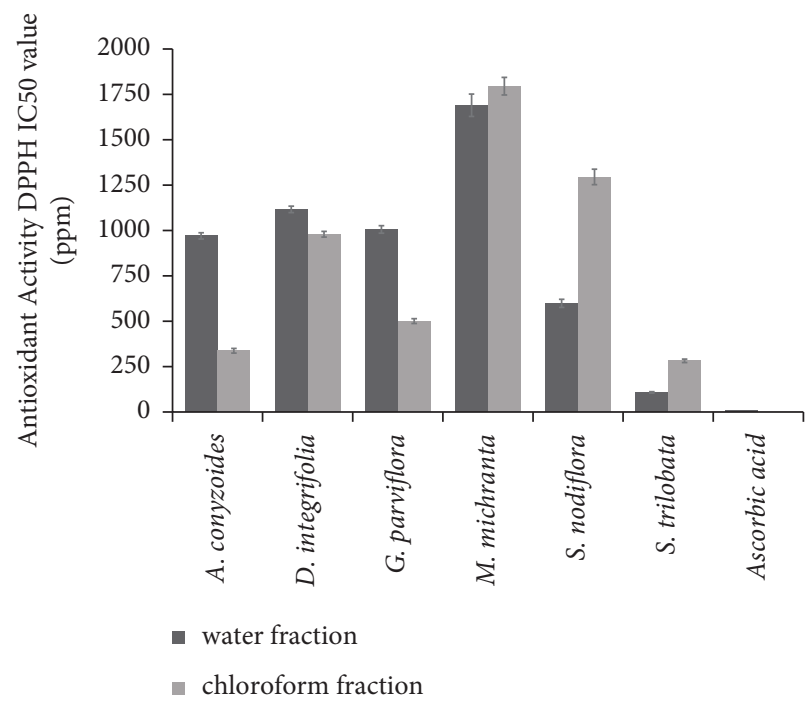

(a)

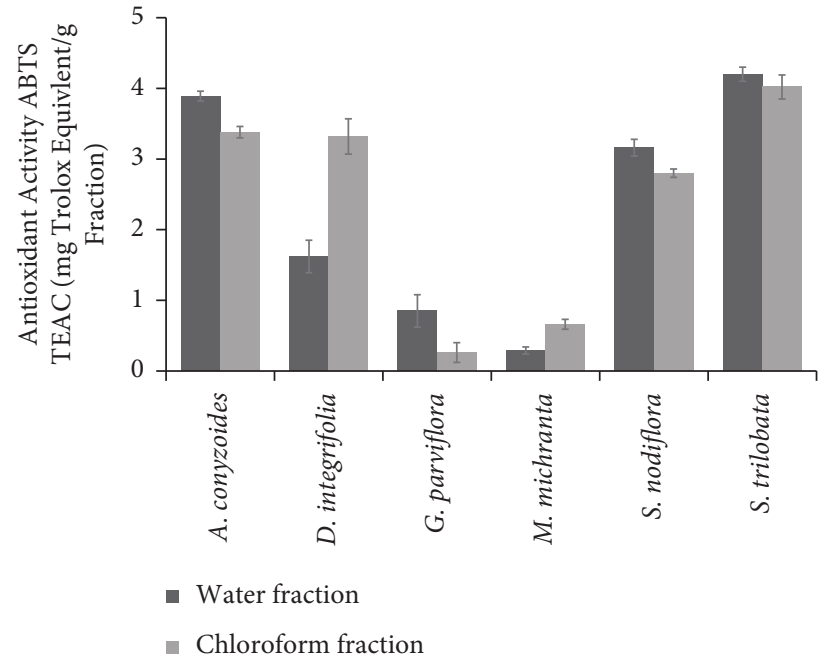

(b)

FIGURE 1: Antioxidant activity of fractions tested in this study. (a) DPPH activity expressed in IC $_{50}$ value (the ability of fractions to scavenge DPPH radicals at 50\%). (b) ABTS method depicted in TEAC (Trolox equivalent antioxidant activity) mg Trolox equivalent/g fraction.

clove, in promoting yeast viability in oxidative stress conditions $[25,33]$. The particular extract with such antioxidant activities may be developed as an antiaging agent. Indeed, a comprehensive review presented the antioxidant properties of various plants, which were applied to promote human skin cell longevity [34]. Therefore, in this study, we analyzed and determined the potential antiaging properties of the Asteraceae plant extracts using yeast as the model organism.

\subsection{Antiaging Activity of Plant Extracts and Fractions.} Based on spot chronological lifespan assays, yeast cell viability can be observed from the spotted assays that emerge on the surface of the plate medium as shown in Figure 2. More spotted assays in the horizontal direction on day 11 indicated high yeast cell viability after 11 days of treatments. All treatments with the extracts and fractions, except those with the chloroform fractions of $A$. conyzoides, were shown to promote yeast cell longevity, compared with the effects of treatment without the plant extracts/fractions (i.e., DMSO alone) (Figure 2). These data were assumed based on the yeast cell viability within the stationary phase on days 7 and 11 , which indicated the chronological age of the yeast cells.

As previously reported, the survival of $S$. pombe cells that were grown in a standard glucose concentration of $3 \%$ decreased by day 7 , and hardly any cells survived beyond day 10 [35]. Therefore, assessment of cell viability within 7 and 11 days may have allowed conceptual perspective on the aging process of $S$. pombe. It is worth noting that prolongation of the yeast lifespan was better after treatment with several extracts/fractions than after calorie restriction treatment; in particular, these were the water extract of D. integrifolia $(10.000 \mathrm{ppm})$ and the chloroform fraction (2505 ppm) and water extract (10000 ppm) of G. parviflora. Therefore, these extracts/fractions have high potential properties as antiaging agents.
Further analysis of yeast cell survival on day 11 using quantitative plate count assay confirmed that all water fractions increased the survival of yeast cells, compared with the effects of control and treatment with DMSO only and without the extracts/fractions (Figure 3(a)). Moreover, treatment with the chloroform fractions of all plants, except A. conyzoides, significantly increased cell survival, compared with the effects of treatment without the extracts/fractions (Figure 3(b)). All the plant water extracts and fractions enhanced the survival rate of yeast cells, but $A$. conyzoides showed insignificant enhancement (Figure 3(c)). Unlike our findings in the qualitative spot assay, we noticed that only the water extract of $D$. integrifolia $(10.000 \mathrm{ppm})$ increased the cell survival at a relatively similar value with that of the calorie restriction treatments (Figure 3(c)). This result suggested that treatment with the water extract of D. integrifolia mimicked calorie restriction-dependent cell longevity in nutrient-rich conditions. Similarly, previous studies revealed that treatment with green tea and resveratrol mimicked calorie restriction conditions, which induce longevity in yeast cells $[36,37]$.

Calorie restriction has been known to slow aging in model organisms and risk or degenerative diseases in mammalian cells [38]. Calorie restriction conditions $(0.5 \%$ glucose) were shown to mediate longevity of the yeast S. cerevisiae grown by decreasing the growth factor signaling of Target of Rapamycin- (TOR-) Sch9 pathways and increasing Nicotinamide Adenine Dinucleotide- (NAD-) dependent deacetylase Sir2 and autophagy [24, 39, 40]. Moreover, reduced TOR signaling in both fission and budding yeasts was shown to enhance mitochondrial respiratory activity and coupling, which subsequently induce both Rim15-mediated ROS detoxification and adaptive mitochondrial ROS signaling, leading to lifespan extension [41]. Therefore, to clarify the involvement of the mitochondria in the mechanism of action of the plant 
TABLE 3: Effect of selected concentration of plant extract/fraction on yeast cells viability.

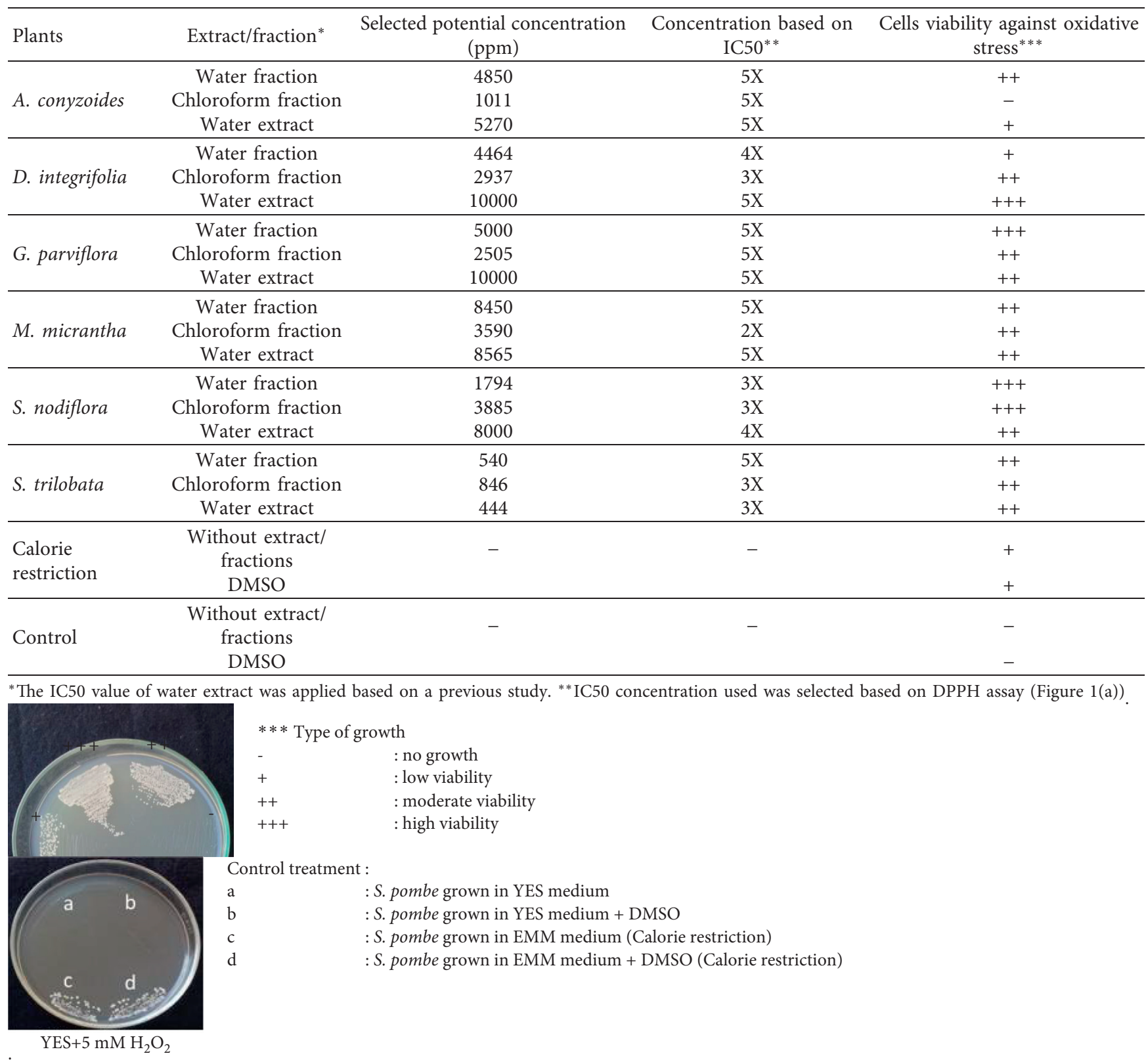

extracts/fractions in extending yeast lifespan, we further tested the effect of the extracts/fractions on the mitochondrial membrane potential.

3.4. Effect of the Plant Extracts/Fractions on Mitochondrial Activity. The mitochondria play important roles in regulating yeast chronological lifespan through adaptive mitochondrial ROS signaling [41]. Such phenomenon has been known to be conserved in yeast, nematode Caenorhabditis elegans, and mice [41-44]. As expected, treatment with the water extract of $D$. integrifolia significantly increased the mitochondrial membrane potential, as shown by the strong fluorescence intensity (Figure 4). Interestingly, in addition to the water extract of $D$. integrifolia, all water extracts of the plant samples induced mitochondrial activity (Figure 4).
Such an increase in mitochondrial activity may further lead to adaptive mitochondrial ROS signaling, which can induce stress tolerance responses. Indeed, this was confirmed by the capability of the yeast cells treated with the water extract in combating strong $\mathrm{H}_{2} \mathrm{O}_{2}$-induced oxidative stress treatments (Table 3).

Interestingly, the water and chloroform fractions of D. integrifolia, M. micrantha, and S. nodiflora enhanced mitochondrial activity, similar to that seen with calorie restriction treatments. Although treatments with these water and chloroform fractions had shorter lifespan extension, compared with that of calorie restriction conditions, they were able to prolong the yeast lifespan, compared with the effect of treatment without the fractions. As reported by a previous study, mitochondria activity and biogenesis induce 


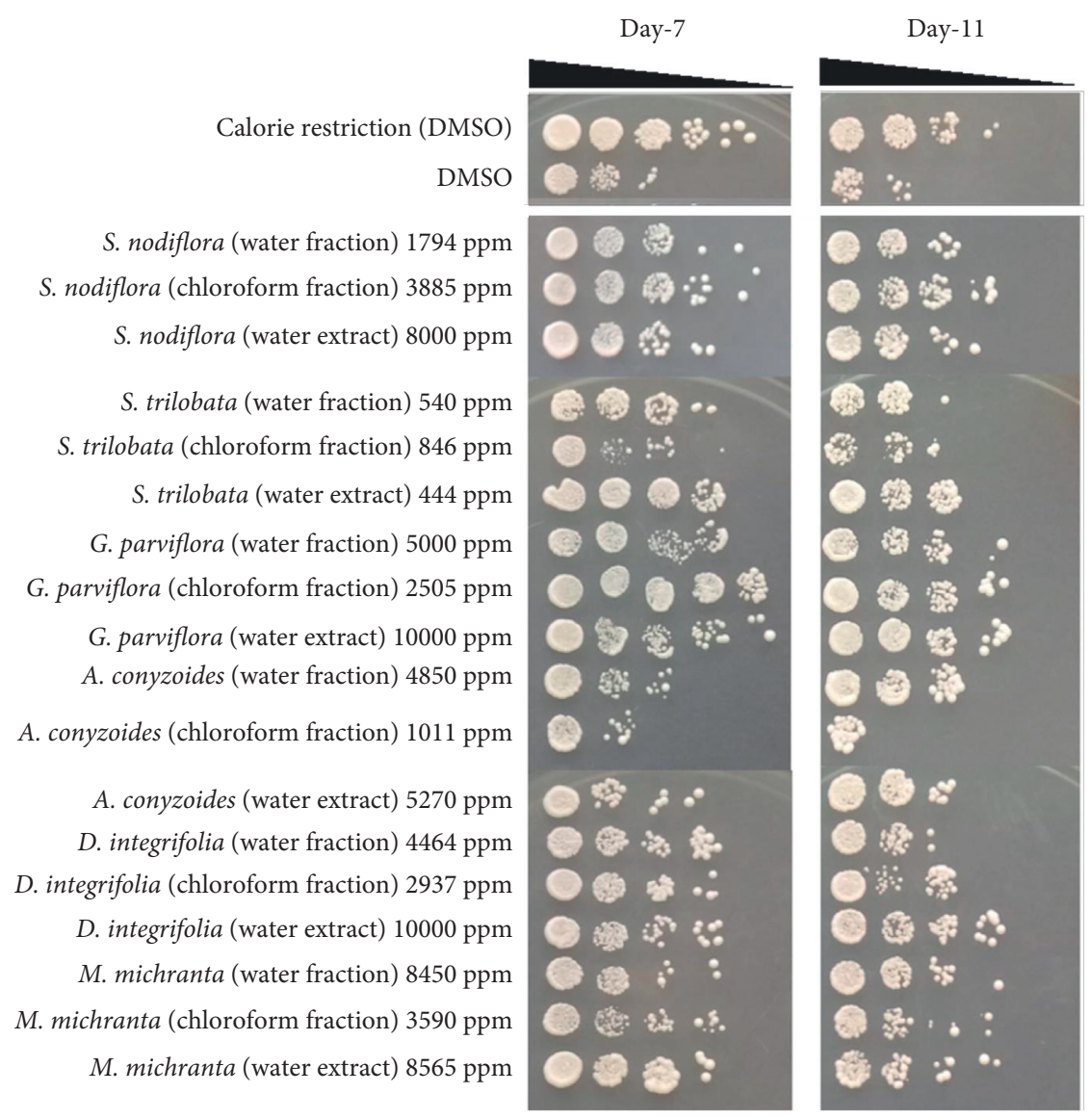

FIGURE 2: Effect of plant extract and fractions on yeast cells viability during stationary phase (chronological aging). Yeast cells were grown in a YES medium containing an optimum concentration of plant extract/fractions as observed previously. Yeast cells grown in EMM medium supplemented with DMSO were designated as calorie restriction for control positive treatment, while yeast cells grown in YES medium supplemented with DMSO only without any extract/fractions addition were used as control. Each culture treatment was then incubated for 7 and 11 days and subjected for spot assay on YES agar. Plates were incubated for three days at $30^{\circ} \mathrm{C}$.

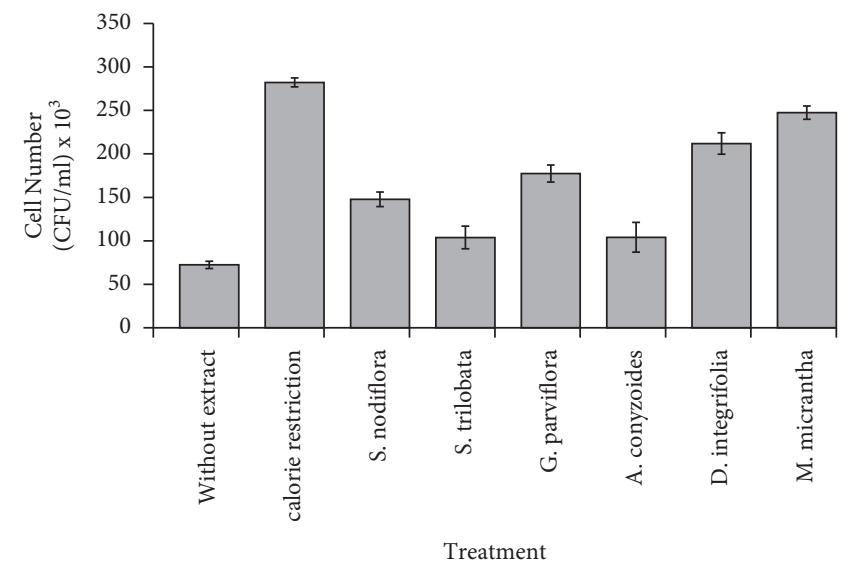

(a)

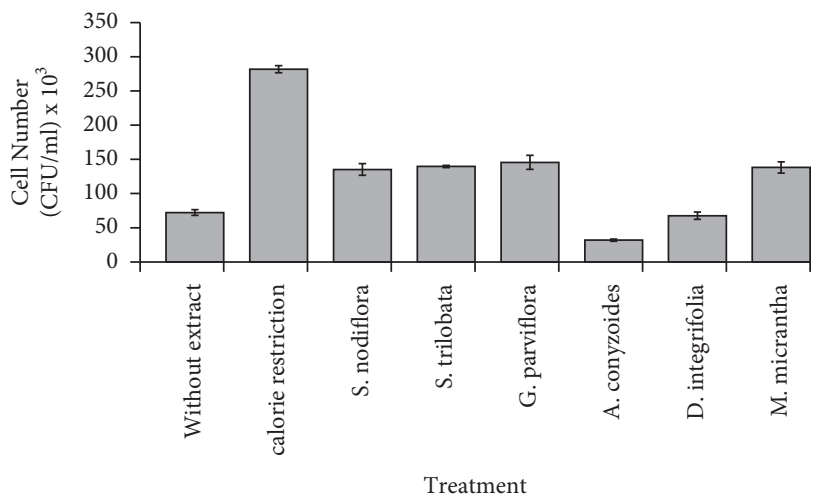

(b)

FIgURE 3: Continued. 


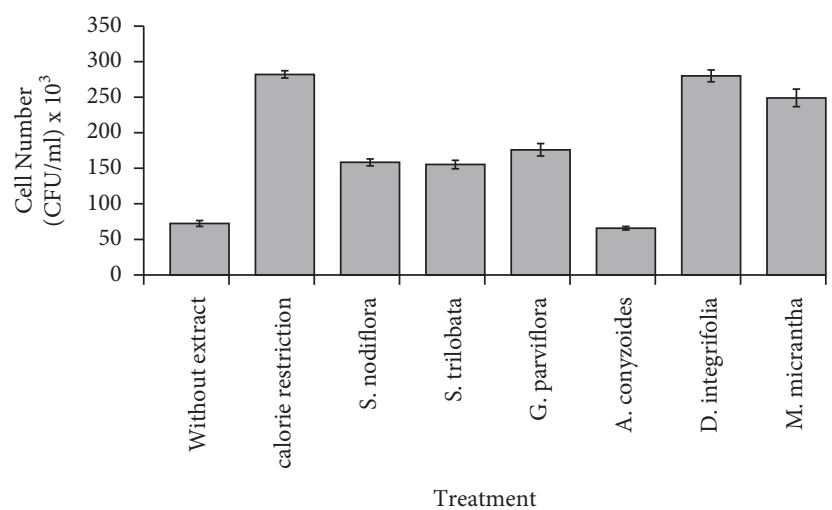

(c)

FIGURE 3: Effect of (a) water fraction, (b) chloroform fraction, and (c) water extract on the cell number of stationary phase-yeast cells (11 days of treatment incubation). Yeast cells were grown in YES medium containing an optimum concentration of plant extract/fractions as observed previously in oxidative stress tolerance assay. Yeast cells grown in EMM medium supplemented with DMSO were designated as calorie restriction for the positive control treatment, while yeast cells grown in YES medium supplemented with DMSO only without any extract/fractions addition were used as control. Each culture treatment was then incubated for 11 days and enumerated for cell concentration based on plate count assay using YES agar. Plates were incubated for three days at $30^{\circ} \mathrm{C}$.

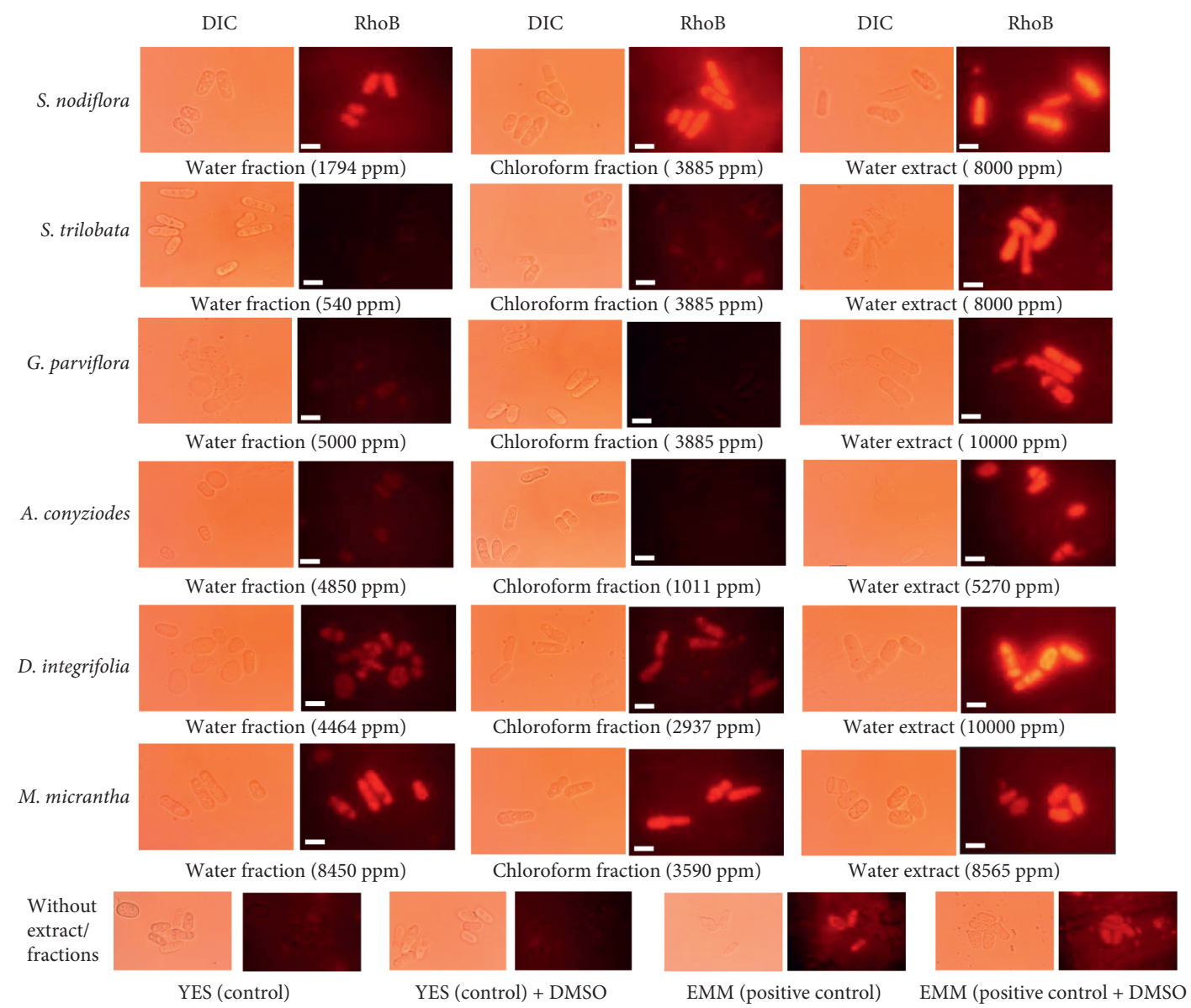

FIGURE 4: Effect of plant extract/fractions on the yeast mitochondrial activity. Yeast was grown in YES broth medium containing various concentrations of extract/fractions. Cells were then harvested and suspended with phosphate buffer. Mitochondrial activity was identified using Rhodamine 123 as a probe under fluorescence microscopy observation. 
cell longevity in yeast [45]. Notably, not all water and chloroform fractions were found to induce mitochondrial activity, nevertheless to its potential antiaging activity. For example, the water and chloroform fractions of A. conyzoides, G. parviflora, and S. trilobata did not induce mitochondrial activity, but they promoted oxidative stress tolerance phenotype and slow aging (Figure 2). These results suggested that the development of oxidative stress tolerance and lifespan extension phenotype of yeast after the application of those particular fractions were independent of the adaptive mitochondrial ROS signaling.

Previous studies indicated that the oxidative stress tolerance of the yeast $S$. pombe was mediated by various pathways, including the stress-activated protein kinase and the cell integrity pathways [44], which mediate the activation of core environmental stress response (CESR) through the key transcription factors Sty1/Atf1 and Pap1 for strong and mild oxidative stress conditions, respectively $[44,46]$. Therefore, we further confirmed the potential induction of CESR genes by the selected plant extracts/fractions.

\subsection{Effect of S. nodiflora Extracts/Fractions on the Expression} of the Gene Involved in Oxidative Stress Tolerance. Among the various genes in the CESR system, the key transcriptional factors gene pap 1 and two genes of the downstream targets, including ctt 1 and sod2, were analyzed in this study. These genes are essential for combating mild oxidative stress, which leads to adaptive oxidative stress response, direct ROS scavenging, and promotion of redox homeostasis in S. pombe $[44,46]$.

For gene expression analysis, we used the extracts and fractions of S. nodiflora. The fraction of S. nodiflora was shown to have quite strong in vitro antioxidant activity and strongly induced mitochondrial activity (Figure 4). The fraction and extract of $S$. nodiflora affected the transcriptional level of the target genes at different patterns. The water fractions increased the expression of all target genes, whereas ctt1 gene was found to be markedly increased 25-fold (Figure 5). The mRNA level of pap1 Transcriptional factor (TF) was increased by the water fraction and water extract, but no significant change in the pap1 mRNA level was detected following treatment with the chloroform fraction. The expression of sod2 gene was upregulated by chloroform fraction and water extract with an expression value of 2-fold compared to the DMSO control (Figure 5). These data indicated that primarily water fractions derived from S. nodiflora induced the pap1-dependent CESR systems in $S$. pombe likely by inducing mitochondrial activity. Such inductions of mitochondrial activity may develop an adaptive mitochondrial ROS signaling and induce the corresponding pap1-dependent CESR system. Indeed, the ctt1 gene encoding catalase protein was significantly transcribed following water fraction. As in yeast $S$. pombe, Ctt1 functions downstream of Tpx1-Pap1 TF and Sty1-Atf1 TF contributed to lifespan extension [47]. Ctt1 is required to protect the yeast cells from the toxicity of $\mathrm{H}_{2} \mathrm{O}_{2}$ radicals and the acquisition of oxidative stress resistance which are likely to increase following fraction-induced high mitochondrial activity [44]. In addition, the sod2 gene for the mitochondrial manganese superoxide dismutase

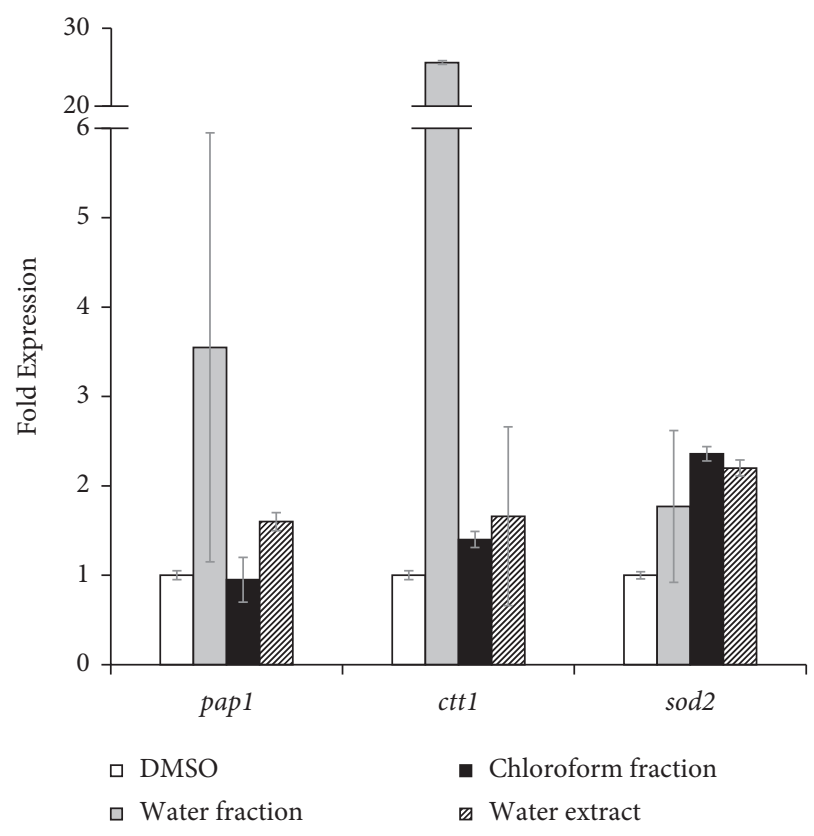

FIgURE 5: Antioxidant-related gene expression (pap1, ctt1, and sod2) of $S$. pombe cells treated with fraction and extract of S. nodiflora based on Reverse Transcriptase PCR analysis. Yeast cells were grown in YES liquid medium supplemented with water fraction, chloroform fraction, and water extract of $S$. nodiflora with each concentration of 1794,3885 , and $8000 \mathrm{ppm}$, respectively. $S$. pombe culture treated with DMSO was used as a control.

was transcripted following chloroform fraction and water extract (Figure 5). As in yeast S. cerevisiae, Sod2 functions downstream of Sch9 contributed to lifespan extension [48]. Sod2 $p$ is required to protect mitochondrial DNA from the toxicity of superoxide anions that are likely to increase following extract-induced high mitochondrial activity [49]. Previous studies described that enhancement of the mitochondrial membrane potential in another yeast S. cerevisiae tor $1 \Delta$ strain resulted in lifespan extension [41, 50]. A similar phenomenon was reported in a prior study, in which exogenous treatment with pyrrolo[1,2-a]pyrazine-1,4-dione that was extracted from marine bacterial culture with Pseudomonas sp. PTR-08 was shown to induce the longevity of the yeast $S$. pombe, likely by modulating the pap1-cttl pathway, in addition to the cell cycle processes [31].

3.6. LC-MS/MS Profile of S. nodiflora Fractions. Results from LC-MS/MS analysis showed that the water and chloroform fractions of $S$. nodiflora have more than 20 compounds. Nevertheless, we identified 6 predicted compounds from each fraction which were dominant and clearly identified as shown in Figures 6(a) and 6(b) (mass spectrum available on Supplementary Figures 1 and 2). The dominant compounds on water fractions, primarily oxyphyllacinol, valine, and sugiol, were reported as having strong antioxidant activity in vitro and in the cell line model [51-53]. Interestingly, sugiol was also reported as having numerous pharmacological properties including antimicrobial, anti-inflammatory, and antivirus along with anticarcinoma [53]. As for chloroform fractions, some 


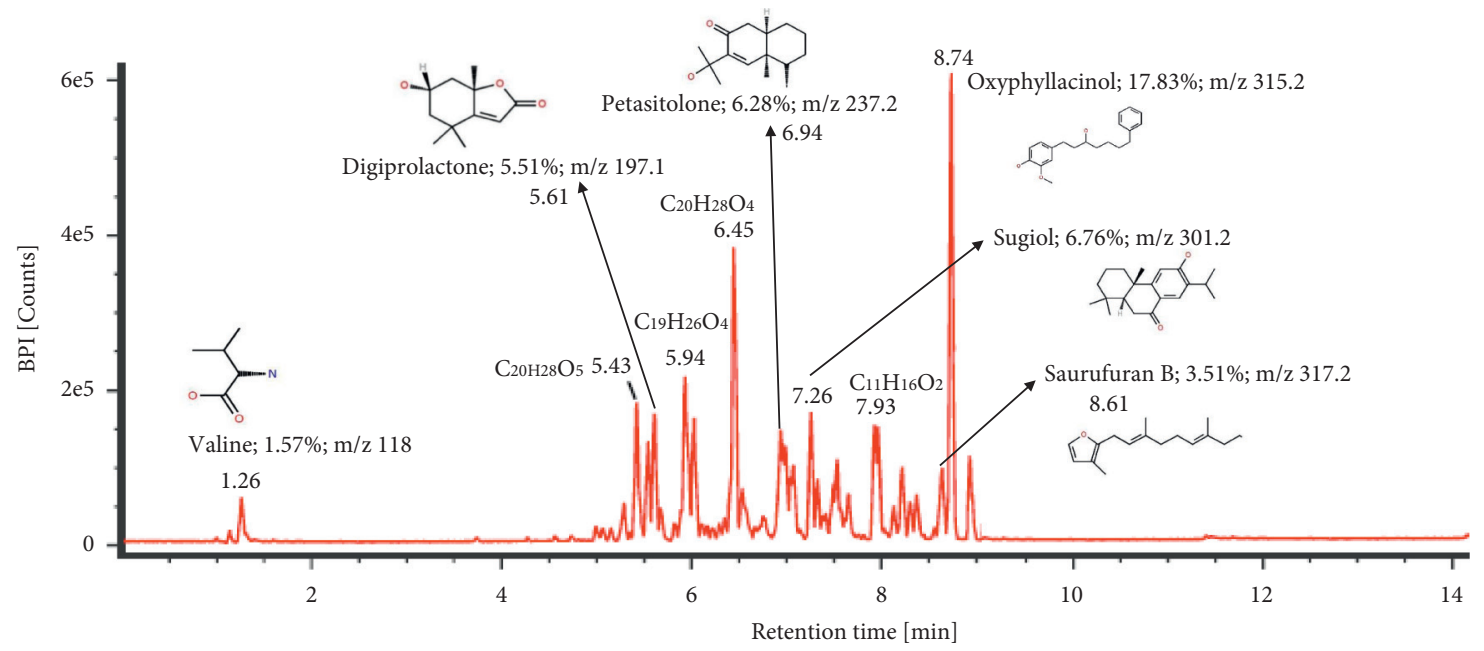

(a)

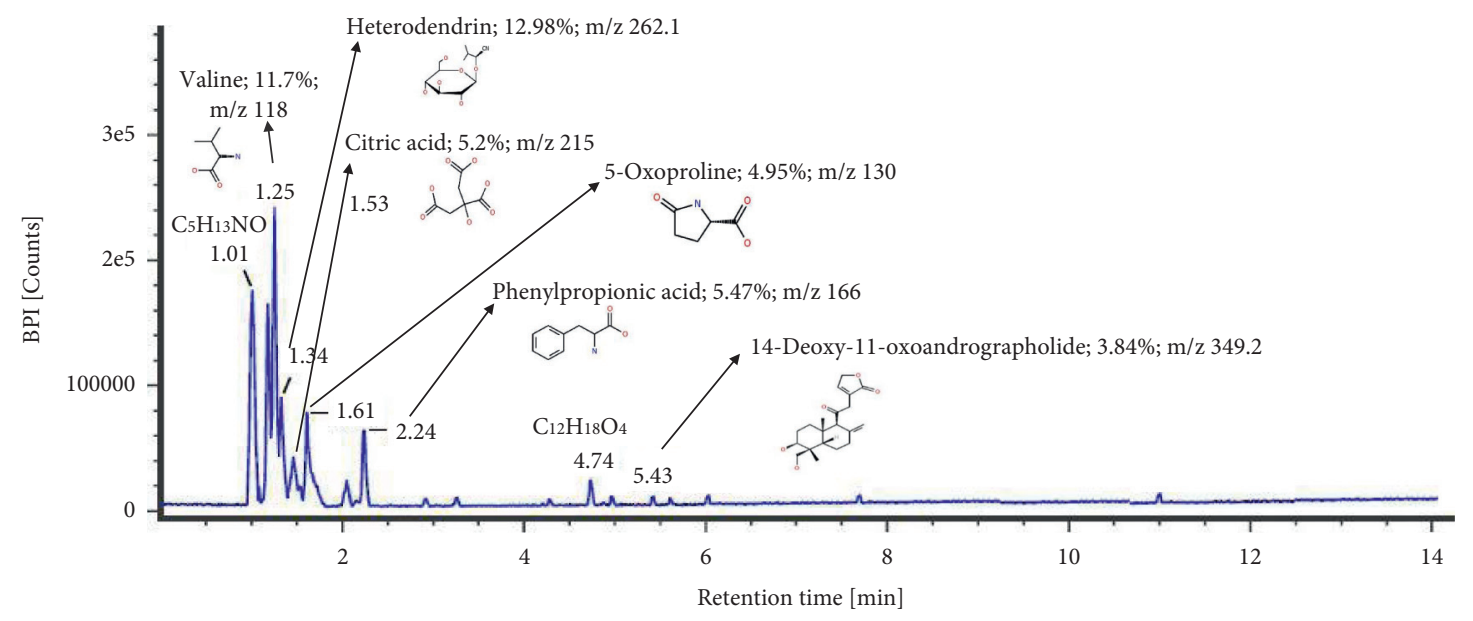

(b)

FIGURE 6: LC-MS/MS profile and predicted compounds of S. nodiflora fractions: (a) water fraction; (b) chloroform fraction.

compounds including 14-deoxy-11-oxoandrographolide, citric acid, and also valine exhibited antioxidant activity in vitro [54-56]. Of note, 14-deoxy-11-oxoandrographolide is a compound belonging to the andrographolide class (diterpenoids), which is also popular with other pharmaceuticals activities, that is, anti-inflammatory and antidiabetic [57, 58]. These results suggested that these dominant compounds especially in water fractions proven with strong in vitro and cell line antioxidant activity might affect the longevity in yeast cells. However, further research should be examined to elucidate each of those potential compounds and retest the corresponding effect in the yeast cells, since reports of the particular activity from those compounds in relation to antioxidant and antiaging mechanisms in yeast cells are still limited.

\section{Conclusions}

The water and chloroform extracts/fractions of Asteraceae plants can alter the cellular functions, induce an intracellular oxidative stress tolerance mechanism, and extend the lifespan of $S$. pombe. Mitochondrial activity increased after treatment with the extracts/fractions, which likely induced mitochondrial adaptive ROS signaling. These results would be essential for the development of the bioactive compounds of Asteraceae plants, particularly as pharmacologic antiaging/antioxidant products.

\section{Data Availability}

All data analyzed during this study are included within the published article.

\section{Conflicts of Interest}

The authors declare that they have no conflicts of interest.

\section{Acknowledgments}

The authors thank Dr. Akhmad Darmawan (Research Center for Chemistry, Indonesian Institute of Sciences (LIPI), Indonesia) for the generous assistance during LCMS/MS analysis. This work was supported by the Ministry of Research and Higher Education of the Republic of Indonesia 
for the research funding given to Irmanida Batubara through the scheme of "Penelitian Dasar Unggulan Perguruan Tinggi” (Grant no. 4160/IT3.I.1/PN/2019).

\section{Supplementary Materials}

Mass spectrum of both S. nodiflora water and chloroform fractions. (Supplementary Materials)

\section{References}

[1] L. A. Pham-Huy, H. He, and C. Pham-Huy, "Free radicals, antioxidants in disease and health," International Journal of Biomedical Sciences, vol. 4, no. 2, pp. 89-96, 2008.

[2] G. Pizzino, N. Irrera, M. Cucinotta et al., "Oxidative stress: harms and benefits for human health," Oxidative Medicine and Cellular Longevity, vol. 2017, pp. 1-13, Article ID 8416763 , 2017.

[3] A. A. Adwas, A. S. I. Elsayed, A. E. Azab, and F. A. Quwaydir, "Oxidative stress and antioxidant mechanisms in human body," Journal of Applied Biotechnology and Bioengineering, vol. 6, no. 1, pp. 43-47, 2019.

[4] E. Birben, U. M. Sahiner, C. Sackesen, S. Erzurum, and O. Kalayci, "Oxidative stress and antioxidant defense," World Allergy Organization Journal, vol. 5, no. 1, pp. 9-19, 2012.

[5] B. L. Tan, M. E. Norhaizan, W.-P.-P. Liew, and H. S. Rahman, "Nutrients and oxidative stress: friend or foe?" Oxidative Medicine and Cellular Longevity, vol. 2018, no. 1162, pp. 1-24, 2018.

[6] H. Zhang and R. Tsao, "Dietary polyphenols, oxidative stress and antioxidant and anti-inflammatory effects," Current Opinion in Food Science, vol. 8, pp. 33-42, 2016.

[7] D. M. Kasote, S. S. Katyare, M. V. Hegde, and H. Bae, "Significance of antioxidant potential of plants and its relevance to therapeutic applications," International Journal of Biological Sciences, vol. 11, no. 8, pp. 982-991, 2015.

[8] Y.-J. Zhang, R.-Y. Gan, S. Li et al., "Antioxidant phytochemicals for the prevention and treatment of chronic diseases," Molecules, vol. 20, no. 12, pp. 21138-21156, 2015.

[9] S. Vijaylakshmi, M. J. Nanjan, and B. Suresh, "In vitro antioxidant activities of Asteraceae plants," Ancient Science of Life, vol. 29, no. 2, pp. 3-6, 2009.

[10] S. Koc, B. S. Isgor, Y. G. Isgor, N. Shomali Moghaddam, and O. Yildirim, "The potential medicinal value of plants from Asteraceae family with antioxidant defense enzymes as biological targets," Pharmaceutical Biology, vol. 53, no. 5, pp. 746-751, 2015.

[11] F. Bakar, Ö. B. Acikara, B. Ergene, S. Nebioğlu, and G. S. Çitoğlu, "Antioxidant activity and phytochemical screening of some Asteraceae plants," Turkish Journal of Pharmaceutical Sciences, vol. 12, no. 2, pp. 123-132, 2015.

[12] I. Faraone, D. K. Rai, L. Chiummiento et al., "Antioxidant activity and phytochemical characterization of senecio clivicolus wedd," Molecules, vol. 23, no. 10, pp. 1-17, 2018.

[13] E. Budiarti, I. Batubara, and A. Ilmiawati, "Potensi beberapa ekstrak tumbuhan asteraceae sebagai antioksidan dan antiglikasi," Jurnal Jamu Indonesia, vol. 4, no. 3, pp. 103-111, 2019.

[14] I. Sadowska-Bartosz and G. Bartosz, "Effect of antioxidants supplementation on aging and longevity," BioMed Research International, vol. 2014, pp. 1-17, Article ID 404680, 2014.

[15] M. B. Wierman and J. S. Smith, "Yeast sirtuins and the regulation of aging," FEMS Yeast Research, vol. 14, no. 1, pp. 73-88, 2014.
[16] M. Watroba and D. Szukiewicz, "The role of sirtuins in aging and age-related diseases," Advances in Medical Sciences, vol. 61, no. 1, pp. 52-62, 2016.

[17] A. Moskalev, A. Aliper, Z. Smit-McBride, A. Buzdin, and A. Zhavoronkov, "Genetics and epigenetics of aging and longevity,” Cell Cycle, vol. 13, no. 7, pp. 1063-1077, 2014.

[18] M. de la Torre-Ruiz, N. Pujol, and V. Sundaran, "Coping with oxidative stress. the yeast model," Current Drug Targets, vol. 16, no. 1, pp. 2-12, 2015.

[19] A. Salminen and K. Kaarniranta, "Regulation of the aging process by autophagy," Trends in Molecular Medicine, vol. 15, no. 5, pp. 217-224, 2009.

[20] A. Schiavi and N. Ventura, "The interplay between mitochondria and autophagy and its role in the aging process," Experimental Gerontology, vol. 56, pp. 147-153, 2014.

[21] S. M. Jazwinski, "Yeast longevity and aging-the mitochondrial connection," Mechanism of Ageing and Development, vol. 126, no. 2, pp. 243-248, 2005.

[22] N. Sun, R. J. Youle, and T. Finkel, "The mitochondrial basis of aging," Molecular Cell, vol. 61, no. 5, pp. 654-666, 2016.

[23] A. Ruetenik and A. Barrientos, "Dietary restriction, mitochondrial function and aging: from yeast to humans," Biochimica et Biophysica Acta (BBA) - Bioenergetics, vol. 1847, no. 11, pp. 1434-1447, 2015.

[24] L. Fontana, L. Partridge, and V. D. Longo, "Extending healthy life span--from yeast to humans," Science, vol. 328, no. 5976, pp. 321-326, 2010 .

[25] A. F. Fauzya, R. I. Astuti, and N. R. Mubarik, "Effect of ethanol-derived clove leaf extract on the oxidative stress response in yeast Schizosaccharomyces pombe," International Journal of Microbiology, vol. 2019, pp. 1-7, Article ID 2145378, 2019.

[26] M. E. Prastya, R. I. Astuti, I. Batubara, H. Takagi, and A. T. Wahyudi, "Natural extract and its fractions isolated from the marine bacterium Pseudoalteromonas flavipulchra STILL-33 have antioxidant and antiaging activities in Schizosaccharomyces pombe," FEMS Yeast Research, vol. 20, no. 3, pp. 1-14, 2020.

[27] M. E. Prastya, R. I. Astuti, I. Batubara, and A. T. Wahyudi, "Antioxidant, antiglycation and in vivo antiaging effects of metabolite extracts from marine sponge-associated bacteria," Indian Journal of Pharmaceutical Sciences, vol. 81, no. 2, pp. 344-353, 2019.

[28] I. Batubara, R. I. Astuti, and M. E. Prastya, "The antiaging effect of active fractions and ent-11 $\alpha$-hydroxy-15-oxo-kaur16-en-19-oic acid isolated from adenostemma lavenia (L.) o. kuntze at the cellular level," Antioxidants, vol. 9, no. 8, pp. 1-14, 2020.

[29] M. E. Prastya, R. I. Astuti, I. Batubara, and A. T. Wahyudi, "Bacillus sp. SAB E-41-derived extract shows antiaging properties via ctt1-mediated oxidative stress tolerance response in yeast Schizosaccharomyces pombe," Asian Pacific Journal of Tropical Biomedicine, vol. 8, no. 11, pp. 533-539, 2018.

[30] A. E. Roux, A. Quissac, P. Chartrand, G. Ferbeyre, and L. A. Rokeach, "Regulation of chronological aging in Schizosaccharomyces pombe by the protein kinases Pka1 and Sck2," Aging Cell, vol. 5, no. 4, pp. 345-357, 2006.

[31] M. E. Prastya, R. I. Astuti, I. Batubara, H. Takagi, and A. T. Wahyudi, "Chemical screening identifies an extract from marine Pseudomonas sp.-PTR-08 as an anti-aging agent that promotes fission yeast longevity by modulating the Pap1ctt1+ pathway and the cell cycle," Molecular Biology Reports, vol. 47, no. 1, pp. 33-43, 2020. 
[32] K. Shinmura, "Effects of caloric restriction on cardiac oxidative stress and mitochondrial bioenergetics: potential role of cardiac sirtuins," Oxidative Medicine and Cellular Longevity, vol. 2013, pp. 1-11, Article ID 528935, 2013.

[33] R. I. A. Sarima, R. I. Astuti, and A. Meryandini, "Modulation of aging in yeast saccharomyces cerevisiae by roselle petal extract (Hibiscus sabdariffa L.)," American Journal of Biochemistry and Biotechnology, vol. 15, no. 1, pp. 23-32, 2019.

[34] S. Jadoon, S. Karim, M. H. H. B. Asad et al., "Anti-aging potential of phytoextract loaded-pharmaceutical creams for human skin cell longetivity," Oxidative Medicine and Cellular Longevity, vol. 2015, pp. 1-17, Article ID 709628, 2015.

[35] J. Stephan, J. Franke, and A. E. Ehrenhofer-Murray, "Chemical genetic screen in fission yeast reveals roles for vacuolar acidification, mitochondrial fission, and cellular GMP levels in lifespan extension," Aging Cell, vol. 12, no. 4, pp. 574-583, 2013.

[36] J. A. Baur, K. J. Pearson, N. L. Price et al., "Resveratrol improves health and survival of mice on a high-calorie diet," Nature, vol. 444, no. 7117, pp. 337-342, 2006.

[37] X. Xie, W. Yi, and P. Zhang, "Green tea polyphenols, mimicking the effects of dietary restriction, ameliorate highfat diet-induced kidney injury via regulating autophagy flux," Nutrients, vol. 9, no. 5, 2017.

[38] L. Fontana, R. M. Adelaiye, A. L. Rastelli et al., "Dietary protein restriction inhibits tumor growth in human xenograft models of prostate and breast cancer," Oncotarget, vol. 4, no. 12, pp. 2451-2461, 2013.

[39] Y. Wang, "Molecular links between caloric restriction and Sir2/SIRT1 activation," Diabetes \& Metabolism Journal, vol. 38, no. 5, pp. 321-329, 2014.

[40] A. Leonov, R. Feldman, A. Piano et al., "Caloric restriction extends yeast chronological lifespan via a mechanism linking cellular aging to cell cycle regulation, maintenance of a quiescent state, entry into a non-quiescent state and survival in the non-quiescent state," Oncotarget, vol. 8, no. 41, pp. 69328-69350, 2017.

[41] Y. Pan, E. A. Schroeder, A. Ocampo, A. Barrientos, and G. S. Shadel, "Regulation of yeast chronological life span by TORC1 via adaptive mitochondrial ROS signaling," Cell Metabolism, vol. 13, no. 6, pp. 668-678, 2011.

[42] W. Yang and S. Hekimi, "A mitochondrial superoxide signal triggers increased longevity in Caenorhabditis elegans," PLoS Biology, vol. 8, no. 12, pp. e1000556-14, 2010.

[43] M. Ristow and S. Schmeisser, "Extending life span by increasing oxidative stress," Free Radical Biology and Medicine, vol. 51, no. 2, pp. 327-336, 2011.

[44] M. A. Papadakis and C. T. Workman, "Oxidative stress response pathways: fission yeast as archetype," Critical Reviews in Microbiology, vol. 41, no. 4, pp. 520-535, 2015.

[45] A. Ocampo, J. Liu, E. A. Schroeder, G. S. Shadel, and A. Barrientos, "Mitochondrial respiratory thresholds regulate yeast chronological life span and its extension by caloric restriction," Cell Metabolism, vol. 16, no. 1, pp. 55-67, 2012.

[46] A. P. Vivancos, M. Jara, A. Zuin, M. Sansó, and E. Hidalgo, "Oxidative stress in Schizosaccharomyces pombe: different $\mathrm{H} 2 \mathrm{O} 2$ levels, different response pathways," Molecular Genetics and Genomics, vol. 276, no. 6, pp. 495-502, 2006.

[47] J. Quinn, V. J. Findlay, K. Dawson et al., "Distinct regulatory proteins control the graded transcriptional response to increasing $\mathrm{H}_{2} \mathrm{O}_{2}$ levels in fission yeast schizosaccharomyces pombe," Molecular Biology of the Cell, vol. 13, no. 3, pp. 805-816, 2002.
[48] P. Fabrizio, L.-L. Liou, V. N. Moy et al., "SOD2 functions downstream of Sch9 to extend longevity in yeast," Genetics, vol. 163, no. 1, pp. 35-46, 2003.

[49] K. M. O’Brien, R. Dirmeier, M. Engle, and R. O. Poyton, "Mitochondrial protein oxidation in yeast mutants lacking manganese- (MnSOD) or copper- and zinc-containing superoxide dismutase (CuZnSOD): evidence that mnsod and cuznsod have both unique and overlapping functions in protecting mitochondrial proteins from oxidative damage," Journal of Biological Chemistry, vol. 279, no. 50, pp. 5181751827, 2004.

[50] N. D. Bonawitz, M. Chatenay-Lapointe, Y. Pan, and G. S. Shadel, "Reduced TOR signaling extends chronological life span via increased respiration and upregulation of mitochondrial gene expression," Cell Metabolism, vol. 5, no. 4, pp. 265-277, 2007.

[51] J. T. Han, S. Y. Lee, Y. H. Lee, and N. I. Baek, "Antioxidative diarylheptanoids from fruits of Alpinia oxyphylla," Food Science and Biotechnology, vol. 16, no. 6, pp. 1060-1063, 2007.

[52] E. Cojocaru, N. Filip, C. Ungureanu, C. Filip, and M. Danciu, "Effects of valine and leucine on some antioxidant enzymes in hypercholesterolemic rats," Health, vol. 06, no. 17, pp. 2313-2321, 2014.

[53] V. K. Bajpai, S. Sonwa, and S. K. Hwang, "Sugiol, a diterpenoid: therapeutic actions and molecular pathways involved," Pharmacological Research, vol. 163, Article ID 105313, 2020.

[54] J. Joselin and S. Jeeva, "Andrographis paniculata: a review of its traditional uses, phytochemistry and pharmacology," Medicinal \& Aromatic Plants, vol. 3, no. 4, pp. 1-15, 2014.

[55] D. Arsenov, M. Zupunski, and M. Borisev, "Exogenously applied citric acid enhances antioxidant defense and phytoextraction of cadmium by Willows (Salix Spp.)," Water, Air, and Soil Pollution, vol. 228, no. 221, pp. 1-12, 2017.

[56] H. J. Jin, J. H. Lee, D. H. Kim et al., "Antioxidative and nitric oxide scavenging activity of branched-chain amino acids," Food Science and Biotechnology, vol. 24, no. 4, pp. 1555-1558, 2015.

[57] G.-F. Dai, J. Zhao, Z.-W. Jiang et al., “Anti-inflammatory effect of novel andrographolide derivatives through inhibition of NO and PGE2 production," International Immunopharmacology, vol. 11, no. 12, pp. 2144-2149, 2011.

[58] M.-J. Lee, Y. K. Rao, K. Chen, Y.-C. Lee, Y.-S. Chung, and Y.-M. Tzeng, "Andrographolide and 14-deoxy-11,12-didehydroandrographolide from Andrographis paniculata attenuate high glucose-induced fibrosis and apoptosis in murine renal mesangeal cell lines," Journal of Ethnopharmacology, vol. 132, no. 2, pp. 497-505, 2010. 